\title{
An interval type-2 fuzzy clustering solution for large-scale multiple-criteria group decision-making problems
}

\author{
Tong Wu, Xin-Wang Liu ${ }^{1}$ \\ School of Economics and Management, Southeast University, Nanjing, 210096, Jiangsu, China
}

\begin{abstract}
In order to deal with the fuzzy large-scale multiple-criteria group decision-making (FLMCGDM) problems, this paper incorporates clustering analysis and information aggregation operator into the problems of large-scale multiple-criteria group decision-making with interval type-2 fuzzy sets (IT2 FSs). The interval type-2 fuzzy equivalence clustering (IT2-FEC) analysis is used to classify decision-makers (DMs) to reduce the dimension of the large-scale DMs in the FLMCGDM problems. The combined weighted geometric averaging (CWGA) operator is extended into the case with IT2 FSs variables, which can take both the importance of individual and its relative position into account. Afterwards, a solution process for the FLMCGDM problems is proposed, in which the new equivalence clustering method and CWGA operator of IT2 FSs is incorporated. Finally, the reasonability and effectiveness of the proposed method are verified by an illustrative example. Compared with other methods, the IT2-FEC analysis can deal with the linguistic variables and produce dynamic clustering results in a more efficient way.
\end{abstract}

Keywords: Interval type-2 fuzzy sets (IT2 FSs); fuzzy large-scale multiple-criteria group decision-making (FLMCGDM); fuzzy equivalence clustering (FEC) analysis; combined weighted geometric averaging (CWGA) operator

\section{Introduction}

Many big decisions in real life usually require a panel of decision makers (DMs) or experts to make, which can be called as group decision-making (GDM) problems. Chen [1] classified the group decision-making problems whose DMs' number is more than 20 into large-scale group decision-making (LGDM) problems. Because of the complexity and vagueness in process of decision-making, time and capital consuming will be huge in the LGDM problems. For a large group that might involve a clustered structure [2], clustering processing is indispensable in group decision-making problems [2-5]. Due to the vagueness, limitation and complexity of decision-making factors, the DMs present preference with specific numerical values may be inaccurate [6]. On the contrary, the linguistic variables that modeled with interval type-2 fuzzy sets (IT2 FSs) can take place of the numerical values expressing DMs' preference in a flexible and unconstrained way [7]. Moreover, the efficient aggregation of data information is a fundamental but important process in decision-making problems [8-13].

Zahir Malgorzata and Alicja [3] have proved that using dynamically generated inseparable clusters in a multi-agent decision-making can improve the efficiency of inference. Liu, et.al [4] adopted the partial

1 Corresponding author. E-mail addresses: tongwu2016@yahoo.com (Tong Wu); xwliu@seu.edu.cn (Xin-Wang Liu). 
binary tree DEA-DA cyclic classification model to present classification of complex multi-attribute large-group decision-making problems. In fact, the most available clustering method in application is the $\mathrm{C}$-means algorithm. The main feature of C-means algorithm is the fact that the clustering numbers should be determined in advance, and the classification results relies mainly on the selection of threshold [14]. However, multiple classification problems in reality always have many aspects that must be considered, which make it hard for DMs to judge classification numbers according to their limited information. Therefore the desired cluster numbers in FCM are difficult to be specified, it is considered that the clustering method with fuzzy equivalence relation (FEC) can generate more natural and more appropriate classification results than FCM [15]. The FEC algorithm has been applied in a variety of fields, such as information retrieval, knowledge discovery, data mining, etc. [16-19].

The fuzzy GDM approaches with linguistic variables have been used widely [20-23]. Due to the fact that the processing of linguistic variables is still rely on the numerical calculation, type-1 fuzzy sets (T1 FSs) has been applied into modeling linguistic variables [24]. However, the nature of membership functions of T1 FSs is crisp values, which means that it is a paradox to use T1 FSs modeling linguistics [25]. Hence, the type-2 fuzzy sets (T2FSs) [26] whose membership functions are defined with T1 FSs has been proposed to model uncertainty instead of T1 FSs. As the most easily understand and calculate form of T2 FSs, IT2 FSs tend to be applied on MCGDM problems [27-29]. Similar to the development of decision-making methods, the clustering analysis method has gone through the transformation from crisp values, type-1 fuzzy sets to type-2 fuzzy sets [30]. As the FEC algorithm can produce dynamic results rather than decide classification numbers in advance, $\mathrm{Wu}$, et.al [31] proposed a clustering analysis method of fuzzy equivalence under the environment of IT2 FSs (IT2 FEC), and verified the efficiency of the proposed method through numerical analysis. With the IT2 FEC algorithm, the large-scale DMs in the LMCGDM problems can be classified into different clusters with similar characteristics to cut the decision-making size on a large degree.

Many efficient aggregation operators [9-13] have been proposed to deal with complex information in multi-attribute group decision-making problems. Liu, et.al [9] used the interval-valued intuitionistic fuzzy weighted average (IVIFWA) operator to aggregate the information modeled with IVIF numbers in large-group decision-making problems. Liu and Liu [11] developed some normal intuitionistic fuzzy aggregation operators based on the ideal of Bonferroni mean, and then proposed a new approach for the multi-attribute GDM with them. With the advantages of the weighted geometric averaging (WGA) operator [32] and the ordered weighted geometric averaging (OWGA) operator [33], $\mathrm{Xu}$ and $\mathrm{Da}$ [8] proposed a combined weighted geometric averaging (CWGA) operator and applied it in combination with WGA operator into decision-making problems. With the advantages of CWGA that it not only consider the individual importance of data information, but also reflect the importance degree of data information's relative position, the CWGA operator was extended into the IT2 FSs form (IT2-CWGA) in this study to deal with decision information.

It is true that the large-scale or complex GDM problems are very commonly encountered in real life, especially in the era of data age. Some reviews have been appeared in [5, 34-39]. However, the studies of 
fuzzy large-scale multi-criteria group decision-making (FLMCGDM) problems are relatively very few. $\mathrm{Xu}$, et.al [37] proposed an improved consensus model for large-group emergency decision-making and developed an approach to manage minority opinions and non-cooperative behaviors. Based on three-dimensional gray relational analysis, Zhu, et.al [5] described a hierarchical clustering approach for a LGDM with double information. Inspired by the idea of principal component analysis (PCA), Liu, et.al [39] presented an interval-valued intuitionistic fuzzy PCA model for complex multi-attribute LGDM. For the FLMCGDM problems, few researchers studied the complexity, vagueness and feature of large volume at the same time. And the PCA model is meant to reduce the dimension of huge subjects, which will destroy the individuation of some DMs. Through the above analysis, we can find that the IT2 FSs can handle the complexity and vagueness in a precise and efficient way. And the IT2 FEC model can reduce the dimension of huge DMs and retain the individuation of them at the same time.

Therefore, this study is focused on the clustering method to deal with FLMCGDM problems where DMs is huge and the preference of DMs are presented by means of linguistic variables instead of crisp numbers. Due to the superiority of IT2 FSs in comparison with T1 FSs, the IT2 FSs are used to present linguistic variables in this research. And the decision information can be processed with IT2-CWGA operator and ranking method of IT2 FSs.

The study is organized as follows: Section 2 gives the basic knowledge about IT2 FSs; Section 3 proposes the new approach to deal with FLMCGDM problems with IT2 FEC method; Section 4 gives an illustrative example to verify the feasibility of the proposed method; Section 5 talks about the conclusion and future work.

\section{Preliminaries}

Some basic knowledge about interval type-2 fuzzy sets is given in this section.

Definition 1. Mendel [40] gave the definition of type-2 fuzzy sets: A type-2 fuzzy set $A$ in the universe of discourse $X$ can be represented by a type- 2 membership function $\mu_{\tilde{\tilde{A}}}$, represented as follows:

$$
A=\left\{\left((x, u), \mu_{\tilde{\tilde{A}}}(x, u)\right) \mid \forall x \in \mathrm{X}, \forall u \in J_{x} \subseteq[0,1]\right\}
$$

In which $0 \leq \mu_{A}(x, u) \leq 1, x$ is the primary variable, $J_{x}$ is the primary membership, $u$ is the secondary variable, $\mu_{\tilde{\tilde{A}}}(x, u)$ is the secondary membership function.

Definition 2. [40] If all the $\mu_{\tilde{\tilde{A}}}(x, u)=1$, then set $A$ is called interval type-2 fuzzy set (IT2 FSs), defined as follows:

$$
A=\int_{X} \int_{u \in J_{x}} 1 /(x, u) \quad J_{x} \subseteq[0,1]
$$

Definition 3. [41] For discrete universes of discourse $X$ and $U$, an embedded type-2 set $A_{e}$ has $M$ elements, where $A_{e}$ contains exactly one element from $J_{x 1}, J_{x 2}, \ldots, J_{x M}$, namely $u_{1}, u_{1}, \ldots, u_{M}$, each with 
its associated secondary grade, namely $f_{x_{1}}\left(u_{1}\right), f_{x_{2}}\left(u_{2}\right), \ldots, f_{x_{N}}\left(u_{M}\right)$ :

$$
A_{e}=\sum_{i=1}^{M}\left[f_{x_{i}}\left(u_{i}\right) / u_{i}\right] / x_{i} \quad u_{i} \in J_{x_{i}} \subseteq U=[0,1]
$$

Definition 4. [41] For discrete universes of discourse $X$ and $U$, an embedded type-1 set $A_{e}$ has $M$ elements, one each from $J_{x 1}, J_{x 2}, \ldots, J_{x M}$, namely $u_{1}, u_{1}, \ldots, u_{M}$ :

$$
A_{e}=\sum_{i=1}^{M} u_{i} / x_{i} \quad u_{i} \in J_{x_{i}} \subseteq U=[0,1]
$$

Set $A_{e}$ is the union of all the primary memberships of set $A_{e}$ in Eq. (3).

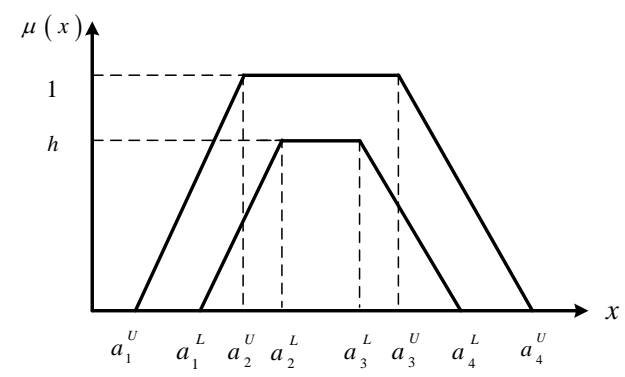

Fig.1. The upper and lower membership function of an trapezoidal IT2 FS

Definition 5. [7] An trapezoidal IT2 FS can be represented in Fig.1, assuming there are two trapezoidal IT2 FSs: $\quad A_{1}=\left(a_{11}^{U}, a_{12}^{U}, a_{13}^{U}, a_{14}^{U}, a_{11}^{L}, a_{12}^{L}, a_{13}^{L}, a_{14}^{L} ; h_{1}\right), A_{2}=\left(a_{21}^{U}, a_{22}^{U}, a_{23}^{U}, a_{24}^{U}, a_{21}^{L}, a_{22}^{L}, a_{23}^{L}, a_{24}^{L} ; h_{2}\right)$. The addition operation between $A_{1}$ and $A_{2}$ is defined as follows:

$$
A_{1} \oplus A_{2}=\left(\left(a_{11}^{U}+a_{21}^{U}, a_{12}^{U}+a_{22}^{U}, a_{13}^{U}+a_{23}^{U}, a_{14}^{U}+a_{24}^{U}\right)\left(a_{11}^{L}+a_{21}^{L}, a_{12}^{L}+a_{22}^{L}, a_{13}^{L}+a_{23}^{L}, a_{14}^{L}+a_{24}^{L} ; \min \left(h_{1}, h_{2}\right)\right)\right)
$$

The multiplication operation between $A_{1}$ and $A_{2}$ is defined as follows:

$$
A_{1} \otimes A_{2} \cong\left(\left(a_{11}^{U} \otimes a_{21}^{U}, a_{12}^{U} \otimes a_{22}^{U}, a_{13}^{U} \otimes a_{23}^{U}, a_{14}^{U} \otimes a_{24}^{U}\right)\left(a_{11}^{L} \otimes a_{21}^{L}, a_{12}^{L} \otimes a_{22}^{L}, a_{13}^{L} \otimes a_{23}^{L}, a_{14}^{L} \otimes a_{24}^{L} ; \min \left(h_{1}, h_{2}\right)\right)\right)
$$

Definition 6. [7] Assuming there is a trapezoidal IT2 FS $A_{i}, A_{i}=\left(a_{i 1}^{U}, a_{i 2}^{U}, a_{i 3}^{U}, a_{i 4}^{U}, a_{i 1}^{L}, a_{i 2}^{L}, a_{i 3}^{L}, a_{i 4}^{L} ; h_{i}\right)$, then the arithmetic operation between $A_{i}$ and the crisp value $k$ are defined as follows:

$$
\begin{aligned}
& k A_{i}=\left(k \times a_{i 1}^{U}, k \times a_{i 2}^{U}, k \times a_{i 3}^{U}, k \times a_{i 4}^{U}, k \times a_{i 1}^{L}, k \times a_{i 2}^{L}, k \times a_{i 3}^{L}, k \times a_{i 4}^{L} ; h_{i}\right) \\
& \frac{1}{k} A_{i}=\left(\frac{1}{k} \times a_{i 1}^{U}, \frac{1}{k} \times a_{i 2}^{U}, \frac{1}{k} \times a_{i 3}^{U}, \frac{1}{k} \times a_{i 4}^{U}, \frac{1}{k} \times a_{i 1}^{L}, \frac{1}{k} \times a_{i 2}^{L}, \frac{1}{k} \times a_{i 3}^{L}, \frac{1}{k} \times a_{i 4}^{L} ; h_{i}\right)
\end{aligned}
$$

\section{The algorithm for LMCGDM problems based on IT2 FEC method}

Based on the theoretical introduction above, a new algorithm for LMCGDM problems based on IT2 FEC method will be proposed. The new method is used to deal with decision-making problems whose DMs' preference is expressed by linguistic variables. In the decision-making process, there are many uncertainties, such as the variety of attributes, the quality of DMs, the unpredictability of environment, etc. 
The uncertainty in decision-making process mainly comes from the DMs whose knowledge and experiences are limited. With so many uncertainties, the dynamic clustering results of DMs that come from IT2 FEC method seem more reasonable. A LMCGDM problem is converted into a typical MCGDM problem after the clustering process. Then the IT2-WGA operator and IT2-CWGA operator are used to deal with the decision information. IT2 FSs will be the main arithmetic elements throughout the whole process of decision-making. The selection of the optimal alternatives relies on the sort of the final calculative results that presented with IT2 FSs. Hence, the defuzzification of IT2 FSs with ranking method is a dispensable step at last.

In order to better present the information processing of FLMCGDM problem with IT2 FEC method and aggregation operators, firstly identify the typical model of FLMCGDM problem. For a FLMCGDM problem, let $D$ be the set of $h$ DMs, where $D=\left\{d_{1}, d_{2}, \ldots, d_{h}\right\}, h \geq 20$ and $F$ be the set of $n$ attributes, where $F=\left\{f_{1}, f_{2}, \ldots, f_{n}\right\}$, and let $X$ be the set of $m$ alternatives, where $X=\left\{x_{1}, x_{2}, \ldots, x_{m}\right\}$. The preferences in decision-making process are presented by linguistic variables and their corresponding IT2 FSs. The DMs' weight $\tilde{\tilde{\mu}}$ and attributes' weight $\tilde{\tilde{w}}$ can be computed with the original decision matrix $\underset{\tilde{A}}{\approx}$. Then, the IT2 FEC method, aggregation operators and ranking method of IT2 FSs will be applied into the following decision-making process.

The interval type-2 fuzzy clustering solution, information aggregation operators and ranking method will be shown in following sections.

\subsection{The fuzzy equivalence clustering method based on IT2 FSs}

In order to reduce the complexity of computation, the fuzzy clustering method based on equivalence relation is applied to divide the large number of DMs in a large group into several groups. Due to the preference of DMs are presented with IT2 FSs, the fuzzy clustering method based on equivalence relation is extended with IT2 FSs (IT2 FEC) [31]. The new clustering method is more flexible and effective to deal with uncertainties and vagueness. And the most important thing is that dynamic results can be obtained with the new method, which reveals the rationality of the IT2 FEC method dealing with uncertainties in FLMCGDM problems.

The nature of the clustering analysis based on fuzzy equivalence relation is that finding more approximate objects to constitute a cluster with the extensibility of the equivalence relation. Therefore, the approximation relation with similarity matrix and the equivalence relation with transitive closure are indispensable parts of the clustering process. In addition, compared with other clustering methods, the dynamic clustering results are the most superiority of the FEC method. The producing principle of the dynamic results is that choose the different clustering coefficient $\beta$ on transitive closure based on the cut set $(\alpha-c u t)$ theory. Finally, make a suitable clustering choice from the dynamic results is also a tough work, it still requires DMs have rich experience and visionary determination out of corresponding algorithms.

The IT2 FEC algorithm mainly has five steps. Firstly, the codebook should be made according to the 
situation and purpose. Then the similarity matrix and transitive closure were computed to get the fuzzy equivalence relationship, and the dynamic clustering results were extracted through the different $\beta$. Finally choose the suitable clustering results according to the realistic situation. The flow chart of the IT2 FEC method is shown in Fig.2.

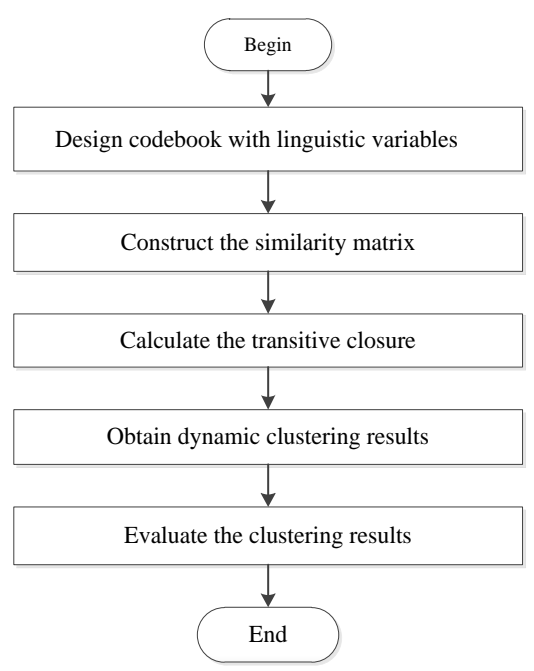

Fig.2. The flow chart for IT2 FEC method

The IT2 FEC algorithm for FLMCGDM problems will be introduced in detail as below.

\section{Step 1. Design codebook and define clustering samples}

A codebook is a simple vocabulary that contains a series of sorted linguistic variables. A codebook should be obtained in different situations with different purposes. Therefore, a new codebook should be designed according to ones' own context since there is no a universal codebook. And define the clustering samples, such as DMs $D=\left\{d_{1}, d_{2}, \ldots, d_{h}\right\}$, attributes $F=\left\{f_{1}, f_{2}, \ldots, f_{n}\right\}$ and alternatives $X=\left\{x_{1}, x_{2}, \ldots, x_{m}\right\}$.

\section{Step 2. Construct the similarity matrix}

DMs give their preference for alternatives with different attributes with suitable linguistic variables from the corresponding codebook. Each linguistic variable can be modeled by the corresponding trapezoidal IT2 FSs according to the codebook. Then the similarity matrix $R=\left[r_{i j}\right]_{h \times h}(1 \leq i, j \leq h)$ between $h$ DMs can be calculated with the IT2 Jaccard similarity method [42].

\section{Step 3. Calculate the transitive closure to obtain the fuzzy equivalence relationship}

The transitive closure $t(R)=\left[t(r)_{i j}\right]_{h \times h}$ of $R$ is the "smallest" fuzzy equivalence relation that contains $R$. There are many methods to compute transitive closure $t(R)$, here, the iterative algorithm of transitive closure was proposed by [15] is adopted.

\section{Step 4. Obtain dynamic clustering results}

The dynamic clustering results were produced with different clustering coefficient $\beta$. Check the value of equivalence matrix, according to cut set $(\alpha-c u t)$ theory, $\beta$ take the numerical values respectively except 0 to get the cut matrices. 


$$
r_{i j}^{*}(\beta)= \begin{cases}1, & r_{i j}^{*} \geq \beta \\ 0, & r_{i j}^{*} \leq \beta\end{cases}
$$

In which, $r_{i j}{ }^{*}$ represents the elements in interval type-2 fuzzy equivalence matrix $R^{*}=t(R)$, $i, j=1,2, \ldots, h$.

\section{Step 5. Evaluate the clustering results}

The clustering numbers emerged unreasonable phenomenon that cluster number is too little or too much when the $\beta$ takes the large or small value on both ends. Choosing the priority clustering result requires that DMs possess rich experience and visionary determination in addition of corresponding algorithms. Assume that the $h$ DMs were classified into $k$ classes with considering the realistic situation, then we can get the new decision group $D^{*}$, where $D^{*}=\left\{G_{1}, G_{2}, \ldots, G_{k}\right\}$.

\subsection{The aggregation operators and the ranking method of IT2 FSs}

The efficient aggregation of data information is a fundamental but important process in decision-making. With the advantages of WGA operator and OWGA operator, Xu and $\mathrm{Da}$ [8] proposed a combined weighted geometric averaging (CWGA) operator and applied it in combination with WGA operator into decision-making problems. The main characteristic of WGA is that obtaining the weighted data firstly according to the importance of each data, and then aggregate the weighted data. And the main characteristic of OWGA is that sort the data according to the order of themselves, and then aggregate them according to their position. As the combination form of WGA and OWGA, a CWGA operator's major characteristic is that it takes the importance degrees of both individual argument and its relative position into account. Depend on the availability and validity of WGA and CWGA operator processing the aggregation information, we extend the WGA and CWGA operator under environment of the IT2 FSs which can be abbreviated as IT2-WGA and IT2-CWGA and applied them into MCLGDM problem.

Firstly, we define the exponent arithmetic operation for trapezoidal IT2 FSs as definition 5.

Definition 7. Assume that there is a trapezoidal IT2 FS $A_{i}=\left(a_{i 1}^{U}, a_{i 2}^{U}, a_{i 3}^{U}, a_{i 4}^{U}, a_{i 1}^{L}, a_{i 2}^{L}, a_{i 3}^{L}, a_{i 4}^{L} ; h_{i}\right)$ and a crisp value $k$, then the exponent operation of $A_{i}$ is defined as follows:

in which,

$$
A_{i}^{k}=\left(\left(a_{i 1}^{U}\right)^{k},\left(a_{i 2}^{U}\right)^{k},\left(a_{i 3}^{U}\right)^{k},\left(a_{i 4}^{U}\right)^{k}\right)\left(\left(a_{i 1}^{L}\right)^{k},\left(a_{i 2}^{L}\right)^{k},\left(a_{i 3}^{L}\right)^{k},\left(a_{i 4}^{L}\right)^{k} ; h_{i}\right),
$$

$$
\begin{aligned}
A_{i}{ }^{k}= & A_{i} \otimes A_{i} \otimes \ldots \otimes A_{i} \\
= & \left(\left(a_{i 1}^{U} \otimes a_{i 1}^{U} \otimes \ldots \otimes a_{i 1}^{U}, a_{i 2}^{U} \otimes a_{i 2}^{U} \otimes \ldots \otimes a_{i 2}^{U}, a_{i 3}^{U} \otimes a_{i 3}^{U} \otimes \ldots \otimes a_{i 3}^{U}, a_{i 4}^{U} \otimes a_{i 4}^{U} \otimes \ldots \otimes a_{i 4}^{U}\right)\right. \\
& \left.\left(a_{i 1}^{L} \otimes a_{i 1}^{L} \otimes \ldots \otimes a_{i 1}^{L}, a_{i 2}^{L} \otimes a_{i 2}^{L} \otimes \ldots \otimes a_{i 2}^{L}, a_{i 3}^{L} \otimes a_{i 3}^{L} \otimes \ldots \otimes a_{i 3}^{L}, a_{i 4}^{L} \otimes a_{i 4}^{L} \otimes \ldots \otimes a_{i 4}^{L} ; \min \left(h_{i}, h_{i}, \ldots, h_{i}\right)\right)\right) \\
= & \left(\left(a_{i 1}^{U}\right)^{k},\left(a_{i 2}^{U}\right)^{k},\left(a_{i 3}^{U}\right)^{k},\left(a_{i 4}^{U}\right)^{k}\right)\left(\left(a_{i 1}^{L}\right)^{k},\left(a_{i 2}^{L}\right)^{k},\left(a_{3}^{L}\right)^{k},\left(a_{i 4}^{L}\right)^{k} ; h_{1}\right)
\end{aligned}
$$

The definition of IT2-WGA and IT2-CWGA operator are shown as follows:

Definition 8. Assume that there is a trapezoidal IT2 FS $A_{i}=\left(a_{i 1}^{U}, a_{i 2}^{U}, a_{i 3}^{U}, a_{i 4}^{U}, a_{i 1}^{L}, a_{i 2}^{L}, a_{i 3}^{L}, a_{i 4}^{L}, h_{i}\right)$, the 
IT2-WGA operator can be shown as following: An interval type-2 fuzzy weighted geometric averaging operator of dimension $n$ is a mapping IT2-WGA $: R^{+^{n}} \rightarrow R^{+}$, which has an index weighted vector $w=\left(w_{1}, w_{2}, \ldots, w_{n}\right)^{T}$ for which $1 \leq p \leq k, w_{j} \in[0,1], \sum_{j=1}^{n} w_{j}=1$, i.e.

$$
\tilde{\widetilde{z}}_{i}^{p}=\operatorname{IT} 2-W G A\left(\tilde{\tilde{b}}_{i 1}^{p}, \tilde{\tilde{b}}_{i 2}^{p}, \ldots, \tilde{\tilde{b}}_{i n}^{p}\right)=\prod_{j=1}^{n}\left(\tilde{\tilde{b}}_{i j}^{p}\right)^{w_{j}}
$$

Definition 9. The IT2-CWGA operator can be shown as following: An interval type-2 fuzzy combined weighted geometric averaging operator of dimension $n$ is a mapping $I T 2-C W G A_{\omega, \lambda}: R^{+^{n}} \rightarrow R^{+}$, which has the associated set of weights $\lambda=\left(\lambda_{1}, \lambda_{2}, \ldots, \lambda_{k}\right)^{T}$ for which $\lambda_{p} \in[0,1], \sum_{p=1}^{k} \lambda_{p}=1$, i.e.,

$$
\tilde{\tilde{z}}_{i}=I T 2-C W G A_{\omega, \lambda}\left(\tilde{\tilde{z}}_{i}^{1}, \tilde{\tilde{z}}_{i}^{2}, \ldots, \tilde{\tilde{z}}_{i}^{k}\right)=\prod_{p=1}^{k}\left(\tilde{\tilde{e}}_{i}^{p}\right)^{\lambda_{p}}
$$

In which, $\tilde{\tilde{e}}_{i}^{p}$ is the $p$ th largest element of the weighted number $\left(\tilde{\tilde{z}}_{i}^{p}\right)^{t \omega_{p}}(i=1,2, \ldots, m ; 1 \leq p \leq k)$, where $\omega_{p}$ is the weighted vector of DMs, and $t$ is the equilibrium factor.

It is easy to prove that the IT2-WGA is a special case of IT2-CWGA, which means that the IT2-CWGA can not only consider the individual importance of data information, but also reflect the importance degree of data information's relative position. Therefore, the IT2-CWGA operator can be effectively applied into decision-making in the real world.

Although the IT2 FSs can ensure the integrity of the information, the comparison operations of decision results are also based on the crisp values. Therefore, the ranking or reduction method of IT2 FSs is indispensable in decision making process under the environment of IT2 FSs.

Wu and Mendel [42] proposed a simple ranking method based on the centroid of IT2 FSs.

Karnik and Mendel [43] extended the centroid definition of T1 FSs into the IT2 FSs as follows:

$$
c(\tilde{\tilde{A}})=\bigcup_{\forall \tilde{A}_{e}} c\left(\tilde{A}_{e}\right)=\left[c_{l}(\tilde{\tilde{A}}), c_{r}(\tilde{\tilde{A}})\right],
$$

where $\bigcup$ is the union operation, $\tilde{A}_{e}$ is the union of all the primary memberships of an embedded type- 2 set $A_{e}$ and

$$
\begin{gathered}
c_{l}(\tilde{\tilde{A}})=\min _{\forall A_{e}} c\left(\tilde{A}_{e}\right) \\
c_{r}(\tilde{\tilde{A}})=\max _{\forall A_{e}} c\left(\tilde{A}_{e}\right) \\
c\left(\tilde{A}_{e}\right)=\frac{\sum_{i=1}^{N} x_{i} \mu_{\tilde{A}_{e}}\left(x_{i}\right)}{\sum_{i=1}^{N} \mu_{\tilde{A}_{e}}\left(x_{i}\right)}
\end{gathered}
$$

in which $c_{l}(\tilde{\tilde{A}}), \quad c_{r}(\tilde{\tilde{A}})$ was obtained from the iterative Karnik and Mendel (KM) algorithm [43]: 


$$
\begin{gathered}
c_{l}(\tilde{\tilde{A}}) \equiv c_{l}(\mathrm{k})=\frac{\sum_{i=1}^{k} x_{i} \overline{u_{\tilde{A}}}\left(x_{i}\right)+\sum_{i=k+1}^{N} x_{i} \underline{u}_{\tilde{\tilde{A}}}\left(x_{i}\right)}{\sum_{i=1}^{k} \overline{u_{\tilde{A}}}\left(x_{i}\right)+\sum_{i=k+1}^{N} \underline{\underline{\tilde{A}}}\left(x_{i}\right)} \\
c_{r}(\tilde{\tilde{A}}) \equiv c_{r}(\mathrm{k})=\frac{\sum_{i=1}^{k} x_{i} \overline{u_{\tilde{A}}}\left(x_{i}\right)+\sum_{i=k+1}^{N} x_{i} \underline{u}_{\tilde{\tilde{A}}}\left(x_{i}\right)}{\sum_{i=1}^{k} \bar{u} \tilde{\tilde{A}}\left(x_{i}\right)+\sum_{i=k+1}^{N} \underline{u}_{\tilde{\tilde{A}}}\left(x_{i}\right)}
\end{gathered}
$$

The centroid-based ranking method is that compute the average centroid for each interval type- 2 fuzzy set $\tilde{\tilde{A}}$,

$$
C\left(A_{i}\right)=\frac{c_{l}\left(A_{i}\right)+c_{r}\left(A_{i}\right)}{2} \quad i=1, \ldots, N
$$

The ranking method has been proved that it satisfies the first four reasonable properties of six reasonable ordering properties of ranking methods for IT2 FSs.

\subsection{The main step of the proposed method}

The proposed decision method is focused on dealing with FLMCGDM problem with IT2 FEC method. The implementation of the clustering process is mainly based on the preference of large-scale DMs. The acquisition of the preference is similar to the traditional decision-making process, firstly, identify the decision-making factors, such as DM set $D$, alternative set $F$ and attribute set $X$. Then construct the original decision matrix $\tilde{\tilde{A}}$ of large-scale DMs. The DMs' weight $\tilde{\tilde{\mu}}$ and attributes' weight $\tilde{\tilde{w}}$ can also be obtained with the preference information processing. The decision matrices are constructed based on the evaluation of alternatives with respect to the attributes. Hence, we can apply the IT2 FEC method into the FLMCGDM problem through some arithmetic process of preference information. There is another traditional decision-making process after the new decision group $D^{*}$ reconstructed with the IT2 FEC method, where $D^{*}=\left\{G_{1}, G_{2}, \ldots, G_{k}\right\}$. Constructed new decision matrix $\tilde{\tilde{B}}$ and weighted matrix $\tilde{\tilde{\omega}}$ for the new decision groups. And then aggregate the decision information with the IT2-WGA operator and the IT2-CWGA operator. The comprehensive attribute value $\tilde{z}_{i}^{p}$ and $\tilde{z}_{i}$ can be computed with these operators respectively. Finally, sort the alternatives with $z_{i}$ which comes from $\widetilde{z}_{i}$ with ranking method of IT2 FSs. The decision process of the FLMCGDM problem with IT2 FEC method can be shown as Fig.3. 


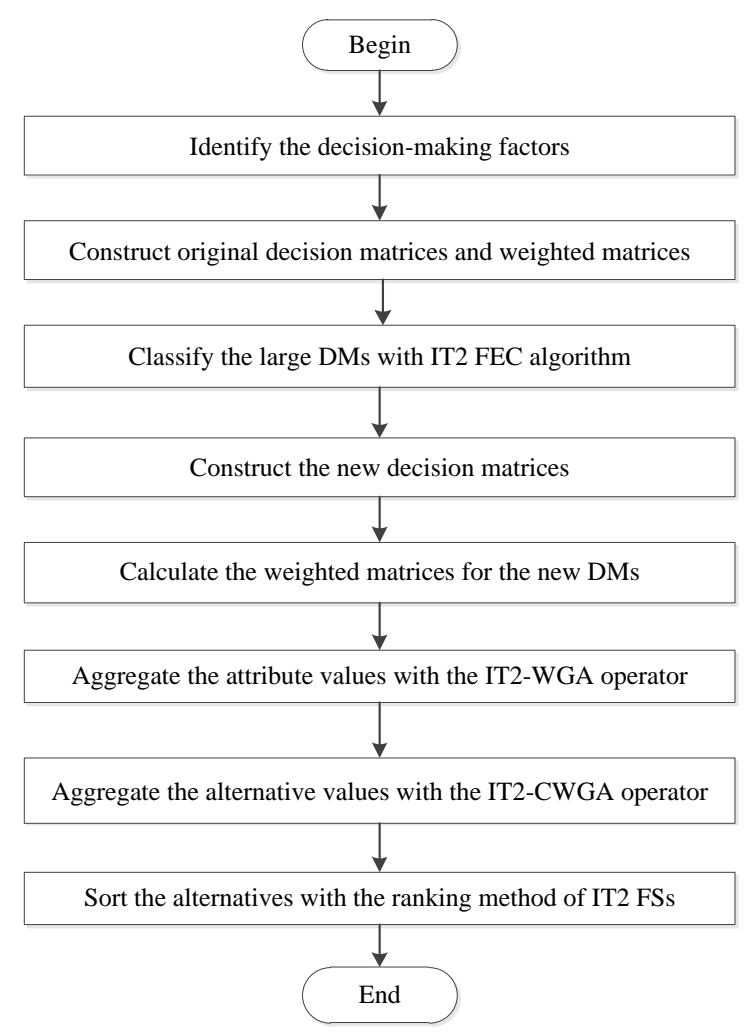

Fig.3. The decision process of the FLMCGDM problem with IT2 FEC method

The detailed steps of the new algorithm for FLMCGDM based on IT2 FEC method are presented as follows:

\section{Step1. Identify the decision-making factors}

For a MCLGDM problem, let $D$ be the set of $h$ DMs, where $D=\left\{d_{1}, d_{2}, \ldots, d_{h}\right\}, h \geq 20$ and $F$ be the set of $n$ attributes, where $F=\left\{f_{1}, f_{2}, \ldots, f_{n}\right\}$, and let $X$ be the set of $m$ alternatives, where $X=\left\{x_{1}, x_{2}, \ldots, x_{m}\right\}$. The preference in decision-making process is presented by linguistic variables and their corresponding IT2 FSs.

\section{Step 2. Construct the original decision matrices and weighted matrices}

DMs give the preference of alternatives with respect to attributes by linguistic variables, and then the original decision matrix $\tilde{\tilde{A}}$ can be constructed. For example, the decision matrix of $p$ th DM can be shown as follows:

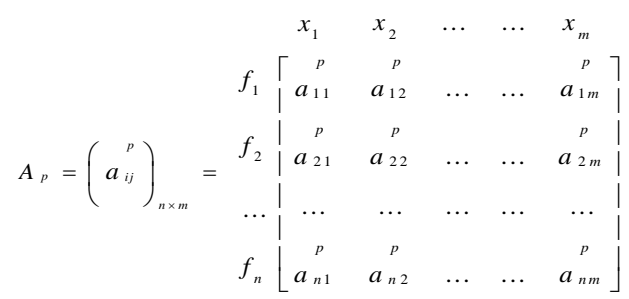

Assume that the attribute weights $W$ are given by DMs: 


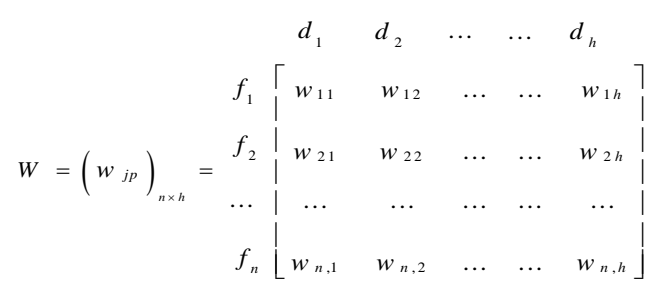

Where $p=1,2, \ldots, h ; j=1,2, \ldots, n$.

Then the weighted vector $\bar{W}$ for attributes can be obtained with the mean value of the weighted matrix of $h$ DMs.

$$
\bar{W}=\left(\tilde{\tilde{w}}_{j}\right)_{1 \times n}
$$

where $\tilde{\tilde{w}}_{j}=\frac{\tilde{\tilde{w}}_{j 1} \oplus \tilde{\tilde{w}}_{j 2} \oplus \ldots \oplus \tilde{\tilde{w}}_{j h}}{h}, \quad \tilde{\tilde{w}}_{j}$ is an IT2 FS, $j=1,2, \ldots, n$.

Sometimes, the interval type-2 fuzzy weighted vector $\tilde{\tilde{w}}$ should be transformed into crisp value $w$ for the convenience of calculation.

\section{Step 3. Classify the large DMs with IT2 FEC algorithm}

Classify the large DMs into several groups according to their different preference with the IT2 FEC algorithm. Based on the original decision matrix $\tilde{\tilde{A}}$ and the weighted vector $\bar{W}$ of attributes, the comprehensive preference $\tilde{\tilde{A}}^{*}$ of DMs can be shown as follows:

Firstly, considering the alternatives $x_{1}, x_{2}, \ldots, x_{m}$ with respect to the attribute $f_{j}$ as a complete individual object $x_{j}$, then the corresponding preference $\tilde{\tilde{a}}_{j}^{p}$ of $p$ th DM on $x_{j}$ is shown as follows:

$$
\tilde{\tilde{\boldsymbol{a}}}_{j}^{p}=\frac{\tilde{\tilde{\boldsymbol{a}}}_{j 1}^{p}+\tilde{\tilde{\boldsymbol{a}}}_{j 2}^{p}+\ldots+\tilde{\tilde{\boldsymbol{a}}}_{j m}^{p}}{m},
$$

where $1 \leq j \leq n ; 1 \leq p \leq h$.

Then, aggregate the preference of $p$ th DM on $x_{j}$ with respect to different attributes to obtain the weighted vector of $p$ th DM.

$$
\tilde{\tilde{a}}_{p}=\tilde{\tilde{w}}_{1} * \tilde{\tilde{a}}_{1}^{p}+\tilde{\tilde{w}}_{2} * \tilde{\tilde{a}}_{2}^{p}+\ldots+\tilde{\tilde{w}}_{n} * \tilde{\tilde{a}}_{n}^{p}
$$

Similarly, weighted vectors of other DMs can also be calculated with the same way. And then the comprehensive preference $\tilde{\tilde{A}}^{*}$ of large-scale DMs can be obtained as $\tilde{\tilde{A}}^{*}=\left\{\tilde{\tilde{a}}_{1}, \tilde{\tilde{a}}_{2}, \ldots, \tilde{\tilde{a}}_{p}, \ldots, \tilde{\tilde{a}}_{h}\right\}$.

Finally, classify the large-scale DMs with the IT2 FEC method. Firstly, the similarity matrix $R=\left[r_{i j}\right]_{h \times h}(1 \leq i, j \leq h)$ between DMs can be computed with the Jaccard method based on the comprehensive preference $\tilde{\tilde{A}}^{*}$, where $r_{i j}=\operatorname{Jaccard}\left(\tilde{\tilde{a}}_{i}, \tilde{\tilde{a}}_{j}\right), 1 \leq i, j \leq h$. Then compute the transitive matrix $t(R)=\left[t(r)_{i j}\right]_{h \times h}$ for $R$. Finally, obtain the dynamic results with the clustering coefficient $\beta$ and choose the suitable clustering result based on the realistic situation. 
Assume that the large-scale decision group-making $D$ is classified as a new decision-making group $D^{*}$, where $D^{*}=\left\{G_{1}, G_{2}, \ldots, G_{k}\right\}, G_{p}$ is a decision-making group whose DMs have the similar preference, which can be seen as a decision-maker. And then the following decision-making process is similar to the traditional methods.

\section{Step 4. Construct the decision matrices for the new decision groups}

According to the clustering results, construct the decision matrix $\tilde{\widetilde{B}}$ for the new decision-making group $D^{*}$ with the mean value of the original decision matrix $\tilde{\widetilde{A}}$. For example, assume that the DM $G_{p}$ contains DM $d_{a}, d_{b}$ and $d_{c}$ in large-scale DMs, the decision matrix $\tilde{\tilde{B}}_{p}$ of the $p$ th decision-maker is shown as follows:

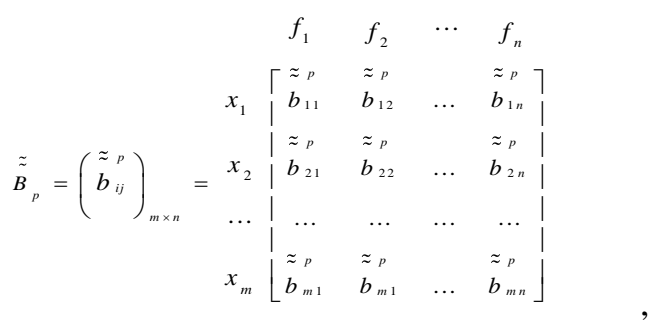

where $\tilde{\tilde{b}}_{i j}^{p}=\frac{\tilde{\tilde{a}}_{i j}^{a}+\tilde{\tilde{b}}_{i j}^{a}+\tilde{\tilde{c}}_{i j}^{a}}{3}, 1 \leq i \leq m, 1 \leq j \leq n, 1 \leq p \leq k$.

\section{Step 5. Construct the weighted matrices for the new DMs}

The weighted matrices for the new DMs should be computed with the clustering results based on the original weighted matrix of the large-scale DMs.

The original weighted matrix $\tilde{\tilde{\mu}}$ of the large-scale DMs can be computed with decision matrix $A$ and the weighted vector $\bar{W}$ for attributes. For example, the weighted matrix $\tilde{\tilde{\mu}}_{p}$ of $p$ th DM can be computed with the decision matrix $\tilde{\tilde{A}}_{p}$ and the weighted vector $\bar{W}$ as follows:

Firstly, the elements in $\tilde{\tilde{A}}_{p}$ can be aggregated with $\bar{W}$ to obtain $\tilde{\tilde{C}}_{p}$ :

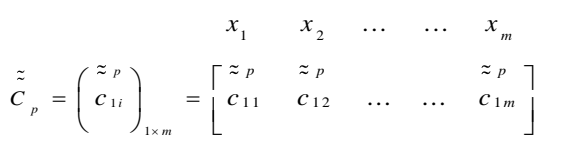

where $\tilde{\tilde{c}}_{1 j}^{p}=\tilde{\tilde{w}}_{1} * \tilde{\tilde{a}}_{1 j}^{p}+\tilde{\tilde{w}}_{2} * \tilde{\tilde{a}}_{2 j}^{p}+\ldots+\tilde{\tilde{w}}_{n} * \tilde{\tilde{a}}_{n j}^{p}, 1 \leq i \leq m$.

Then, the geometric mean of each row $r_{C_{p}}$ is calculated as follows:

$$
r_{C_{p}}=\left(\tilde{\tilde{c}}_{11}^{p} \otimes \tilde{\tilde{c}}_{12}^{p} \otimes \ldots \otimes \tilde{\tilde{c}}_{1 m}^{p}\right)^{1 / m}
$$

Other elements can be calculated as the same way, and then the weighted matrix $\mu_{p}$ is showed as follows:

$$
\tilde{\tilde{\mu}}_{p}=r_{C_{p}} /\left(r_{C_{1}} \oplus r_{C_{2}} \oplus \ldots \oplus r_{C_{h}}\right)
$$


Finally, the original weighted matrix $\tilde{\tilde{\mu}}$ can be obtained, where $\tilde{\tilde{\mu}}=\left\{\tilde{\tilde{\mu}}_{1}, \tilde{\tilde{\mu}}_{2}, \ldots, \tilde{\tilde{\mu}}_{p}, \ldots, \tilde{\tilde{\mu}}_{h}\right\}$.

According to the original weighted matrix $\tilde{\tilde{\mu}}$, the weighted vector $\tilde{\tilde{\tilde{\omega}}}=\left\{\tilde{\tilde{\omega}}_{1}, \tilde{\tilde{\omega}}_{2}, \ldots, \tilde{\tilde{\omega}}_{k}\right\}$ of the new DMs $D^{*}=\left\{G_{1}, G_{2}, \ldots, G_{k}\right\}$ can be computed with the clustering results. For example, assume that the DM $G_{p}$ contains DM $d_{a}, d_{b}$ and $d_{c}$ in large-scale DMs, the weighted vector $\tilde{\tilde{\omega}}_{p}$ of the DM $G_{p}$ can be computed as follows: $\tilde{\tilde{\omega}}_{p}=\left(\tilde{\tilde{c}}_{a}+\tilde{\tilde{c}}_{b}+\tilde{\tilde{c}}_{c}\right) / 3,1 \leq a, b, c \leq h$. Sometimes, the interval type-2 fuzzy weighted vector $\tilde{\tilde{\omega}}$ should be transformed into crisp value $\omega$ for the convenience of calculation.

\section{Step 6. Aggregate the attribute values with the IT2-WGA operator}

Considering the individual importance degree of alternatives, the attribute values can be aggregated with the IT2-WGA operator. For example, the attribute values on $i$ th line in decision matrix $\tilde{\tilde{B}}_{p}$ of the $p$ th decision-maker can be aggregated with the IT2-WGA operator, which can obtain the comprehensive attribute value $\tilde{\tilde{z}}_{i}^{p}$ of $i$ th alternative.

$$
\tilde{\widetilde{z}}_{i}^{p}=I T 2-W G A\left(\tilde{\tilde{b}}_{i 1}^{p}, \tilde{\tilde{b}}_{i 2}^{p}, \ldots, \tilde{\tilde{b}}_{i n}^{p}\right)=\prod_{j=1}^{n}\left(\tilde{\tilde{\tilde{b}}}_{i j}^{p}\right)^{w_{j}},
$$

where $1 \leq j \leq n, 1 \leq p \leq k, w_{j}$ is obtained from $\tilde{\tilde{w}}_{j}$ with the ranking method of IT2 FSs.

\section{Step 7. Aggregate the alternative values with the IT2-CWGA operator}

Considering the relative position of alternatives, the alternative values can be aggregated with the IT2-CWGA operator. For example, the group comprehensive attribute value $\tilde{z}_{i}$ of alternative $x_{i}$ can be obtained with the aggregation of $\tilde{\widetilde{z}}_{i}^{p}$,

$$
\tilde{\tilde{z}}_{i}=I T 2-C W G A_{\omega, \lambda}\left(\tilde{\tilde{z}}_{i}^{1}, \tilde{\tilde{z}}_{i}^{2}, \ldots, \tilde{\tilde{z}}_{i}^{k}\right)=\prod_{p=1}^{k}\left(\tilde{\tilde{e}}_{i}^{p}\right)^{\lambda_{p}},
$$

Where $\lambda_{p}$ is the associated set of weights $\lambda=\left(\lambda_{1}, \lambda_{2}, \ldots, \lambda_{k}\right)^{T}, \tilde{\tilde{e}}_{i}^{p}$ is the $p$ th biggest element of the weighted value $\left(\tilde{z}_{i}^{p}\right)^{t \omega_{p}}(i=1,2, \ldots, m ; 1 \leq p \leq k)$, where $\omega_{p}$ is the weighted vector of DMs, and $t$ is the equilibrium factor.

\section{Step 8. Sort the alternatives with the ranking method of IT2 FSs}

Due to the ranking value $\tilde{z}_{i}$ is an IT2 FS, the ranking method of IT2 FSs is used to obtain the comparable values $z_{i}(1 \leq i \leq m)$ of $\tilde{z}_{i}$. Finally, sort the alternatives with the crisp value $z_{i}$.

\section{Numerical examples}

In this section, we use an example to illustrate the interval type-2 fuzzy large-scale multiple-criteria group decision-making process of the proposed method.

Assume that there are thirty DMs $d_{1}, d_{2}, \ldots, d_{30}$ of a software company to hire a senior manager and assume that there are three alternatives $x_{1}, x_{2}, x_{3}$ and five attributes $f_{1}, f_{2}, f_{3}, f_{4}, f_{5}$, where $f_{1}$ is 
"Emotional Steadiness", $f_{2}$ is "Oral Communication Skill", $f_{3}$ is "Personality", $f_{4}$ is "Past Experience", $f_{5}$ is "Self-Confidence". Let $D$ be the set of DMs, where $D=\left\{d_{1}, d_{2}, \ldots, d_{30}\right\}$, and let $X$ be the set of alternatives, where $X=\left\{x_{1}, x_{2}, x_{3}\right\}$, and let $F$ be the set of attributes, where $F=\left\{f_{1}, f_{2}, f_{3}, f_{4}, f_{5}\right\}$. Assume that thirty DMs use the linguistic variables shown in Table 1 to represent the weights of the five attributes and to represent the evaluating values of the alternatives with respect to different attributes.

\begin{tabular}{cc} 
Table 1. Linguistic terms and their corresponding interval type-2 fuzzy sets \\
\hline Linguistic variables & Trapezoidal interval type-2 fuzzy sets \\
\hline Very Low (VL) & $(0,0,0,0.1 ; 1)(0,0,0,0.05 ; 0.9)$ \\
Low (L) & $(0,0.1,0.2,0.3 ; 1)(0.05,0.12,0.18,0.25 ; 0.9)$ \\
Medium Low (ML) & $(0.1,0.3,0.4,0.5 ; 1)(0.2,0.32,0.38,0.45 ; 0.9)$ \\
Medium (M) & $(0.3,0.5,0.6,0.7 ; 1)(0.4,0.52,0.58,0.65 ; 0.9)$ \\
Medium High (MH) & $(0.5,0.7,0.8,0.9 ; 1)(0.6,0.72,0.78,0.85 ; 0.9)$ \\
High (H) & $(0.7,0.8,0.9,1 ; 1)(0.75,0.82,0.88,0.95 ; 0.9)$ \\
Very High (VH) & $(0.9,1,1,1 ; 1)(0.95,1,1,1 ; 0.9)$ \\
\hline
\end{tabular}

Step1. Identify the decision-making factors

We have identified the sets of DMs $D$, the sets of alternatives $X$ and the sets of corresponding attributes $F$ that shown as above.

\section{Step 2. Constructed the original decision matrices and weighted matrices}

DMs give the preference of alternatives with respect to attributes by linguistic variables, then the original decision matrix $\tilde{\tilde{A}}$ can be constructed. For example, the decision matrix $\tilde{\tilde{A}}_{p}$ of $p$ th DM can be constructed as follows:

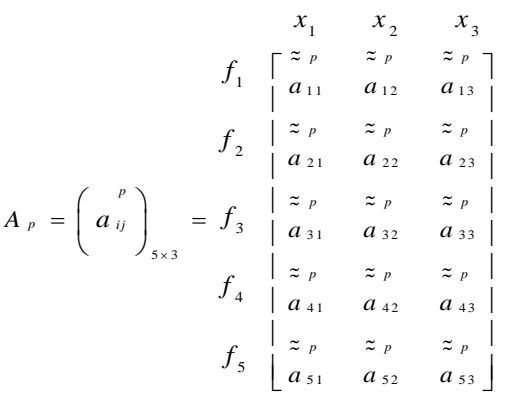

Assume that the attribute weights $W$ are given by DMs:

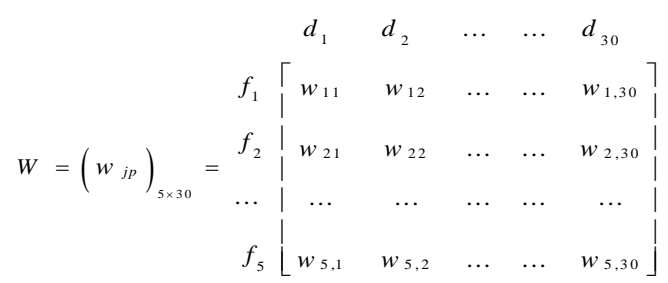

Where $p=1,2, \ldots, 30 ; j=1,2, \ldots, 5$.

Then the weighted vector $\bar{W}$ for attributes can be obtained with the mean value of the weighted 
matrix of $30 \mathrm{DMs}$.

$$
\bar{W}=\left(\tilde{\widetilde{w}}_{j}\right)_{1 \times 31}
$$

where $\tilde{\tilde{w}}_{j}=\frac{\tilde{\tilde{w}}_{j 1} \oplus \tilde{\tilde{w}}_{j 2} \oplus \ldots \oplus \tilde{\tilde{w}}_{j 30}}{30}, \quad \tilde{\tilde{w}}_{j}$ is an IT2 FS, $j=1,2, \ldots, 5$.

The weighted matrices of attributes that presented by IT2 FSs can be found in Table 2. And the crisp value of $\tilde{\tilde{w}}_{j}$ can be calculated by the ranking method of IT2 FSs, the normalized weight $w_{j}(1 \leq j \leq 5)$ of five attributes are $0.19,0.22,0.19,0.22$ and 0.18 respectively.

Table 2. The weighted matrices of attributes with IT2 FSs

\begin{tabular}{cc}
\hline Attributes' weight & Interval type-2 fuzzy sets \\
\hline$\tilde{\tilde{w}}_{1}$ & $(0.61,0.76,0.84,0.92 ; 1)(0.68,0.77,0.82,0.88 ; 0.9)$ \\
$\tilde{\tilde{w}}_{2}$ & $(0.76,0.88,0.93,0.98 ; 1)(0.82,0.89,0.92,0.95 ; 0.9)$ \\
$\tilde{\tilde{w}}_{3}$ & $(0.63,0.77,0.83,0.90,1)(0.72,0.78,0.82,0.87 ; 0.9)$ \\
$\tilde{\tilde{w}}_{4}$ & $(0.74,0.87,0.92,0.97 ; 1)(0.81,0.88,0.91,0.95 ; 0.9)$ \\
$\tilde{\tilde{w}}_{5}$ & $(0.54,0.70,0.78,0.87 ; 1)(0.62,0.71,0.77,0.83 ; 0.9)$ \\
\hline
\end{tabular}

Step 3. Classify the large DMs with IT2 FEC algorithm

Classify the large DMs into several groups according to their different preference with the IT2 FEC algorithm. Based on the original decision matrix $\widetilde{\widetilde{A}}$ and the weighted vector $\bar{W}$ of attributes, the comprehensive preference $\tilde{\tilde{A}}^{*}$ of DMs can be obtained, where $\tilde{\tilde{A}}^{*}=\left\{\tilde{\tilde{a}}_{1}, \tilde{\tilde{a}}_{2}, \ldots, \tilde{\tilde{a}}_{p}, \ldots, \tilde{\tilde{a}}_{h}\right\}$. Then classify the large DMs according to their comprehensive preference $\tilde{\tilde{A}}^{*}$ with the IT2 FEC algorithm. Finally, a new decision-making group $D^{*}$ is obtained through a series of data processing, where $D^{*}=\left\{G_{1}, G_{2}, G_{3}, G_{4}, G_{5}\right\}, G_{1}$ contains $\left\{d_{1}, d_{2}, d_{3}, d_{4}, d_{7}, d_{8}, d_{10}, d_{11}, d_{13}, d_{14}, d_{17}, d_{19}, d_{22}, d_{23}, d_{24}, d_{26}, d_{29}, d_{30}\right\}, G_{2}$ contains $\left\{d_{5}, d_{15}\right\}, G_{3}$ contains $\left\{d_{20}, d_{27}\right\}, G_{4}$ contains $\left\{d_{6}, d_{12}, d_{18}, d_{25}, d_{28}\right\}, G_{5}$ contains $\left\{d_{9}, d_{16}, d_{21}\right\}$, $G_{p}$ is a decision-making group whose DMs have the similar preference, which can be seen as a decision-maker.

Step 4. Construct the decision matrices for the new decision groups

According to the clustering results, the decision matrices for new decision groups can be constructed with the mean value of the original decision matrix $\tilde{\widetilde{A}}$ of thirty DMs about five attributes. For example, the decision matrix $\tilde{\tilde{B}}_{1}$ of the decision group $G_{1}$ can be constructed as follows.

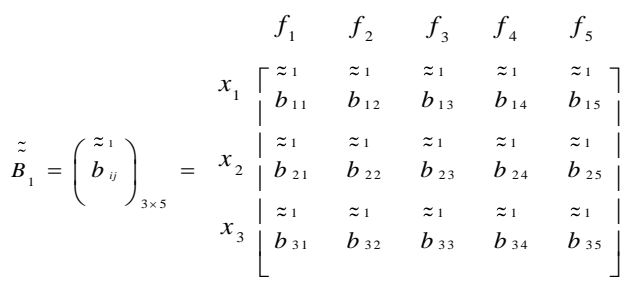


Where $\quad \tilde{\tilde{b}}_{i j}^{1}=\left(\tilde{\tilde{a}}_{i j}^{1}+\tilde{\tilde{a}}_{i j}^{2}+\tilde{\tilde{a}}_{i j}^{3}+\tilde{\tilde{a}}_{i j}^{4}+\tilde{\tilde{a}}_{i j}^{7}+\tilde{\tilde{a}}_{i j}^{8}+\tilde{\tilde{a}}_{i j}^{10}+\tilde{\tilde{a}}_{i j}^{11}+\tilde{\tilde{a}}_{i j}^{13}+\tilde{\tilde{a}}_{i j}^{14}+\tilde{\tilde{a}}_{i j}^{17}+\tilde{\tilde{a}}_{i j}^{19}+\tilde{\tilde{a}}_{i j}^{22}+\tilde{\tilde{a}}_{i j}^{23}+\tilde{\tilde{a}}_{i j}^{24}+\tilde{\tilde{a}}_{i j}^{26}+\tilde{\tilde{a}}_{i j}^{29}+\tilde{\tilde{a}}_{i j}^{30}\right) / 18$, $1 \leq i \leq 3,1 \leq j \leq 5,1 \leq p \leq 5$.

The decision matrices of decision group $G_{1}$ are shown in Table 3.

Table 3. The decision matrices of decision-maker $G_{1}$

\begin{tabular}{lllllll}
\hline$G_{1}$ & \multicolumn{1}{c}{$\mathrm{f} 1$} & \multicolumn{1}{c}{$\mathrm{f} 2$} & \multicolumn{1}{c}{$\mathrm{f} 3$} & $\mathrm{f} 4$ & $\mathrm{f} 5$ \\
\hline $\mathrm{x} 1$ & $(0.67,0.80,0.87,0.94 ; 1)$ & $(0.74,0.87,0.92,0.97 ; 1)$ & $(0.60,0.74,0.82,0.89 ; 1)$ & $(0.74,0.87,0.92,0.97 ; 1)$ & $(0.54,0.70,0.79,0.88 ; 1)$ \\
& $(0.73,0.81,0.86,0.91 ; 0.9)$ & $(0.81,0.88,0.91,0.95 ; 0.9)$ & $(0.67,0.75,0.80,0.86 ; 0.9)$ & $(0.81,0.88,0.91,0.95 ; 0.9)$ & $(0.62,0.72,0.77,0.83 ; 0.9)$ \\
x2 & $(0.60,0.76,0.82,0.89 ; 1)$ & $(0.53,0.69,0.78,0.86 ; 1)$ & $(0.41,0.57,0.67,0.77 ; 1)$ & $(0.53,0.68,0.77,0.85 ; 1)$ & $(0.49,0.64,0.71,0.78 ; 1)$ \\
& $(0.68,0.77,0.81,0.86 ; 0.9)$ & $(0.61,0.71,0.76,0.82 ; 0.9)$ & $(0.49,0.59,0.65,0.72 ; 0.9)$ & $(0.61,0.70,0.75,0.81 ; 0.9)$ & $(0.56,0.65,0.70,0.75 ; 0.9)$ \\
x3 & $(0.49,0.65,0.74,0.84 ; 1)$ & $(0.59,0.73,0.81,0.89 ; 1)$ & $(0.57,0.72,0.79,0.86 ; 1)$ & $(0.53,0.67,0.76,0.85 ; 1)$ & $(0.53,0.69,0.78,0.87 ; 1)$ \\
& $(0.57,0.67,0.73,0.79 ; 0.9)$ & $(0.66,0.74,0.79,0.85 ; 0.9)$ & $(0.64,0.73,0.77,0.83 ; 0.9)$ & $(0.60,0.69,0.74,0.81 ; 0.9)$ & $(0.61,0.71,0.76,0.82 ; 0.9)$ \\
\hline
\end{tabular}

Step 5. Construct the weighted matrices

According to the clustering results, the original weight vector $\tilde{\tilde{\mu}}$ of large-scale DMs can be computed with decision matrix $A$ and the weighted vector $\bar{W}$ for attributes. The weight vector $\tilde{\tilde{\omega}}$ of the new decision groups can be computed with the value mean of the original weight vector $\tilde{\tilde{\mu}}$. The weighted matrices of DMs that presented by IT2 FSs are shown in Table 4, and the crisp value of DMs' weights can be calculated with the ranking method of IT2 FSs, the normalized weight vector $\omega_{j}(1 \leq j \leq 5)$ of five DMs are $0.20,0.22,0.22,0.18$ and 0.18 respectively.

Table 4. The weighted matrices of DMs with IT2 FSs

\begin{tabular}{cc}
\hline DMs & Interval type-2 fuzzy sets \\
\hline$\tilde{\tilde{\omega}}_{1}$ & $(1.88,2.86,3.43,4.06 ; 1)(2.36,2.96,3.31,3.75 ; 0.9)$ \\
$\tilde{\tilde{\omega}}_{2}$ & $(2.22,3.20,3.77,4.41 ; 1)(2.70,3.30,3.66,4.10 ; 0.9)$ \\
$\tilde{\tilde{\omega}}_{3}$ & $(2.12,3.13,3.70,4.33 ; 1)(2.62,3.23,3.59,4.02 ; 0.9)$ \\
$\tilde{\tilde{\omega}}_{4}$ & $(1.68,2.62,3.19,3.83 ; 1)(2.14,2.72,3.07,3.52 ; 0.9)$ \\
$\tilde{\tilde{\omega}}_{5}$ & $(1.67,2.60,3.15,3.77 ; 1)(2.12,2.70,3.04,3.47 ; 0.9)$ \\
\hline
\end{tabular}

Step 6. Aggregate the attributes value with the IT2-WGA operator

The attributes value on $i$ th line in decision matrix $\tilde{\tilde{B}}_{1}$ of the decision group $G_{1}$ can be aggregated

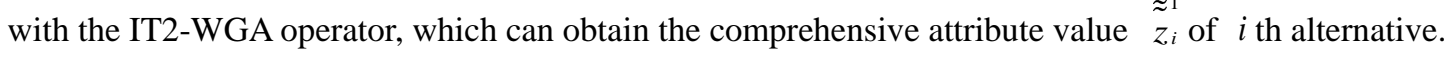

$$
\tilde{\tilde{z}}_{i}^{1}=I T 2-W G A\left(\tilde{\tilde{b}}_{i 1}^{1}, \tilde{\tilde{b}}_{i 2}^{1}, \tilde{\tilde{b}}_{i 3}^{1}, \tilde{\tilde{b}}_{i 4}^{1}, \tilde{\tilde{b}}_{i 5}^{1}\right)=\prod_{j=1}^{5}\left(\tilde{\tilde{b}}_{i j}^{1}\right)^{w_{j}},
$$

where $1 \leq j \leq 5, w_{j}$ is obtained from $\tilde{\tilde{w}}_{j}$ with type-reduction method of IT2 FSs. The comprehensive attribute value $\tilde{\tilde{z}}_{1}^{1}$ of alternative $x_{1}$ from decision-maker $G_{1}$ is computed as follows: 


$$
\begin{aligned}
\tilde{z}_{1}^{1} & =[(0.67,0.80,0.87,0.94 ; 1)(0.73,0.81,0.86,0.91 ; 0.9)]^{0.19} \\
& *[(0.74,0.87,0.92,0.97 ; 1)(0.81,0.88,0.91,0.95 ; 0.9)]^{022} \\
& *[(0.60,0.74,0.82,0.89 ; 1)(0.67,0.75,0.80,0.86 ; 0.9)]^{0.19} \\
& *[(0.74,0.87,0.92,0.97 ; 1)(0.81,0.88,0.91,0.95 ; 0.9)]^{0.22} \\
& *[(0.54,0.70,0.79,0.88 ; 1)(0.62,0.72,0.77,0.83 ; 0.9)]^{0.18} \\
& =(0.66,0.80,0.87,0.93 ; 1)(0.73,0.81,0.85,0.90 ; 0.9)
\end{aligned}
$$

Similarly, other comprehensive attribute value can be obtained as following shows:

$$
\begin{aligned}
& \tilde{\tilde{z}}_{2}^{1}=(0.51,0.67,0.75,0.83 ; 1)(0.59,0.68,0.73,0.79 ; 0.9) \\
& \tilde{\tilde{z}}_{3}^{1}=(0.54,0.69,0.78,0.86 ; 1)(0.62,0.71,0.76,0.82 ; 0.9) \\
& \tilde{\tilde{z}}_{1}^{2}=(0.61,0.77,0.84,0.91 ; 1)(0.70,0.79,0.83,0.88 ; 0.9) \\
& \tilde{\tilde{z}}_{2}^{2}=(0.72,0.83,0.90,0.96 ; 1)(0.78,0.85,0.89,0.93 ; 0.9) \\
& \tilde{\tilde{z}}_{3}^{2}=(0.66,0.79,0.88,0.97 ; 1)(0.73,0.81,0.86,0.93 ; 0.9) \\
& \tilde{\tilde{z}}_{1}^{3}=(0.69,0.83,0.89,0.95 ; 1)(0.76,0.84,0.88,0.92 ; 0.9) \\
& \tilde{\tilde{z}}_{2}^{3}=(0.53,0.69,0.78,0.87 ; 1)(0.61,0.71,0.76,0.83 ; 0.9) \\
& \tilde{\tilde{z}}_{3}^{3}=(0.70,0.83,0.90,0.97 ; 1)(0.77,0.84,0.89,0.94 ; 0.9) \\
& \tilde{\tilde{z}}_{1}^{4}=(0.65,0.79,0.86,0.92 ; 1)(0.72,0.81,0.84,0.89 ; 0.9) \\
& \tilde{\tilde{z}}_{2}^{4}=(0.47,0.62,0.72,0.82 ; 1)(0.54,0.64,0.70,0.77 ; 0.9) \\
& \tilde{\tilde{z}}_{3}^{4}=(0.36,0.53,0.62,0.72 ; 1)(0.45,0.55,0.60,0.67 ; 0.9) \\
& \tilde{\tilde{z}}_{1}^{s}=(0.59,0.74,0.82,0.90 ; 1)(0.67,0.76,0.80,0.86 ; 0.9) \\
& \tilde{\tilde{z}}_{2}^{s}=(0.51,0.65,0.72,0.80 ; 1)(0.58,0.67,0.71,0.76 ; 0.9) \\
& \tilde{\tilde{z}}_{3}^{s}=(0.38,0.54,0.63,0.73 ; 1)(0.46,0.56,0.62,0.68 ; 0.9)
\end{aligned}
$$

Step 7. Aggregate the alternatives value with the IT2-CWGA operator

The group comprehensive attribute value $\tilde{\tilde{z}}_{1}$ of alternative $x_{1}$ can be obtained with the aggregation of $\tilde{\tilde{z}}_{1}^{1}$,

$$
\tilde{\tilde{z}}_{1}=I T 2-C W G A_{\lambda, \omega}\left(\tilde{\tilde{z}}_{1}^{1}, \tilde{\tilde{z}}_{1}^{2}, \tilde{\tilde{z}}_{1}^{3}, \tilde{\tilde{z}}_{1}^{4}, \tilde{z}_{1}^{5}\right)=\prod_{l=1}^{5}\left(\tilde{\tilde{e}}_{1}^{\prime}\right)^{\omega_{t}},
$$

where $\tilde{\tilde{e}}_{1}^{\prime}$ is the $l$ th biggest element of the weighted value $\left(\tilde{\tilde{z}}_{1}^{1}\right)^{t \omega_{1}},\left(\tilde{\tilde{z}}_{1}^{2}\right)^{t \omega_{2}},\left(\tilde{\tilde{z}}_{1}^{3}\right)^{t \omega_{3}},\left(\tilde{\tilde{z}}_{1}^{4}\right)^{t \omega_{4}},\left(\tilde{\tilde{z}}_{1}^{5}\right)^{t \omega_{5}}$, $\omega$ is the corresponding weights of DMs, $t=5$, assuming the associated set of index weight $\lambda=(0.1,0.2,0.3,0.1,0.3)^{T}$.

$\left(\tilde{z}_{1}^{1}\right)^{5 \omega_{1}}=(0.66,0.80,0.87,0.93 ; 1)(0.73,0.81,0.85,0.90 ; 0.9)$

$\left(\tilde{z}_{1}^{2}\right)^{5 \omega_{2}}=(0.59,0.75,0.83,0.90 ; 1)(0.67,0.77,0.81,0.87 ; 0.9)$

$\left(\tilde{\tilde{z}}_{1}^{3}\right)^{5 \omega_{3}}=(0.67,0.81,0.88,0.95 ; 1)(0.74,0.82,0.87,0.92 ; 0.9)$

$\left(\tilde{z}_{1}^{4}\right)^{5 \omega_{4}}=(0.68,0.81,0.87,0.93 ; 1)(0.75,0.82,0.86,0.90 ; 0.9)$ 
$\left(\tilde{\tilde{z}}_{1}^{5}\right)^{5 \omega_{5}}=(0.62,0.76,0.84,0.91 ; 1)(0.70,0.78,0.82,0.87 ; 0.9)$

$\left(\tilde{\tilde{z}}_{2}^{1}\right)^{5 \omega_{1}}=(0.51,0.67,0.75,0.83 ; 1)(0.59,0.68,0.73,0.79 ; 0.9)$

$\left(\tilde{\tilde{z}}_{2}^{2}\right)^{5 \omega_{2}}=(0.69,0.82,0.89,0.96 ; 1)(0.76,0.83,0.88,0.93 ; 0.9)$

$\left(\tilde{z}_{2}^{3}\right)^{5 \omega_{3}}=(0.49,0.67,0.76,0.86 ; 1)(0.58,0.69,0.74,0.81 ; 0.9)$

$\left(\tilde{\tilde{z}}_{2}^{4}\right)^{5 \omega_{4}}=(0.50,0.65,0.74,0.83 ; 1)(0.58,0.67,0.73,0.79 ; 0.9)$

$\left(\tilde{\tilde{z}}_{2}^{5}\right)^{5 \omega_{5}}=(0.54,0.68,0.75,0.82 ; 1)(0.61,0.69,0.74,0.78 ; 0.9)$

$\left(\tilde{\tilde{z}}_{3}^{1}\right)^{5 \omega_{1}}=(0.54,0.69,0.78,0.86 ; 1)(0.62,0.71,0.76,0.82 ; 0.9)$

$\left(\tilde{\tilde{z}}_{3}^{2}\right)^{5 \omega_{2}}=(0.63,0.77,0.87,0.97 ; 1)(0.70,0.79,0.85,0.92 ; 0.9)$

$\left(\tilde{\tilde{z}}_{3}^{3}\right)^{5 \omega_{3}}=(0.67,0.81,0.89,0.97 ; 1)(0.75,0.83,0.87,0.93 ; 0.9)$

$\left(\tilde{z}_{3}^{4}\right)^{5 \omega_{4}}=(0.40,0.56,0.65,0.74 ; 1)(0.48,0.58,0.63,0.70 ; 0.9)$

$\left(\tilde{\tilde{z}}_{3}^{5}\right)^{5 \omega_{5}}=(0.41,0.57,0.66,0.75 ; 1)(0.50,0.59,0.65,0.71 ; 0.9)$

$\tilde{\tilde{z}}_{1}=[(0.67,0.81,0.88,0.95 ; 1)(0.74,0.82,0.87,0.92 ; 0.9)]^{0.1}$

$*[(0.68,0.81,0.87,0.93 ; 1)(0.75,0.82,0.86,0.90 ; 0.9)]^{0.2}$

$*[(0.66,0.80,0.87,0.93 ; 1)(0.73,0.81,0.85,0.90 ; 0.9)]^{0.3}$

$*[(0.62,0.76,0.84,0.91 ; 1)(0.70,0.78,0.82,0.87 ; 0.9)]^{0.1}$

$*[(0.59,0.75,0.83,0.90 ; 1)(0.67,0.77,0.81,0.87 ; 0.9)]^{0.3}$

$=(0.64,0.79,0.85,0.92 ; 1)(0.72,0.80,0.84,0.89 ; 0.9)$

Similarly, we can get the group comprehensive attribute value $\tilde{\tilde{z}}_{2}, \tilde{\tilde{z}}_{3}$ of alternative $x_{2}$ and $x_{3}$ :

$$
\begin{aligned}
& \tilde{\tilde{z}}_{2}=(0.56,0.70,0.79,0.87 ; 1)(0.63,0.72,0.77,0.83 ; 0.9) \\
& \tilde{\tilde{z}}_{3}=(0.52,0.68,0.77,0.86 ; 1)(0.60,0.70,0.75,0.81 ; 0.9)
\end{aligned}
$$

\section{Step 8. Sort the alternatives}

According to the centroid ranking method of Wu and Mendel [37], $\tilde{\tilde{z}}_{1}, \tilde{\tilde{z}}_{2}, \tilde{\tilde{z}}_{3}$ can be transformed into crisp values $z_{1}, z_{2}, z_{3}$, where $z_{1}=0.80, z_{2}=0.73, z_{3}=0.71$. Sort the alternatives with the $z_{i}(1 \leq i \leq 3)$, then $x_{1}$ is the optimal alternative.

According to the ranking result of alternatives, the feasibility of the given approach is proved with the illustrative example. Firstly, the $30 \mathrm{DMs}$ in the fuzzy large-scale multiple-criteria group decision-making (FLMCGDM) problem were classified into 5 clusters with the IT2-FEC algorithm under the environment of interval type-2 fuzzy sets. Therefore, the dimension of the DMs was reduced and the FLMCGDM problem was transformed into a typical and traditional multiple-criteria group decision-making (MCGDM) problem. Then, the transformational MCGDM problem under the environment of high degree of uncertainty can be processed with the interval type-2 fuzzy aggregation operators and the corresponding defuzzified method. Finally, the ranking results were obtained.

The given approach has the following advantages:

(1) According to the similar preference information of large-scale DMs, the proposed approach can 
deal with the large-scale group decision-making problems with the IT2-FEC algorithm.

(2) The given approach can handle the uncertainty and vagueness with linguistic variables that modeled by IT2 FSs in decision-making process.

(3) And the applied aggregation operator (IT2-CWGA operator) for processing the decision information in the proposed method can take the importance degrees of both individual argument and its relative position into account.

(4) In addition, the processing of decision information with IT2 FSs in decision-making process can ensure the integrity of information.

However, the given approach still has disadvantage, some indispensable process of type-reduction and defuzzification on IT2 FSs can also cause the loss of information in some ways.

\section{Conclusion and further study}

Due to the fact that the decision making problems are very complex when the number of DMs (DMs) is huge, this study is focused on the clustering method to deal with fuzzy large-scale multiple-criteria group decision-making (FLMCGDM) problems. The preference information in FLMCGDM problems are better presented by means of linguistic variables, which can be modeled by IT2 FSs due to the fact that the accuracy and flexibility of IT2 FSs are better than T1 FSs. Firstly, clustering method was applied into the classification of large-scale DMs. Compared with the complexity of FCM, the clustering analysis method of fuzzy equivalence under the environment of IT2 FSs (IT2 FEC) is more appropriate to classify the large DMs in FLMCGDM problems. Then, the combined weighted geometric averaging (CWGA) operator takes the importance degrees of both individual argument and its relative position into account, and it is extended into IT2 FSs (IT2-CWGA) to integrate the decision-making information. The decision-making information that is processed with IT2-CWGA operator is expressed with linguistic variables of IT2 FSs, which can ensure the integrity of information and avoid the loss of information. Finally, the main steps of the new proposed method which contains IT2 FEC algorithm and IT2-CWGA operator were given, and the reasonability and effectiveness of the developed method are verified by an illustrative example. In conclusion, the proposed method not only considers the uncertainty and vagueness with proper expression methods in decision-making problems under the environment of IT2 FSs, but also solves the complex problems of large-scale DMs in FLMCGDM problems.

The feasibility and effectiveness of the proposed method have been verified by an illustrative example. However, there are still many improvements and developments can be fulfilled. These include the improvement of algorithms and the exploration of application area. The main research topics of further study are shown as blow:

(1) The operational method and ranking method of IT2 FSs can be redefined in a more reasonable way.

(2) Variety of information aggregation operators should be presented and suitable operators should be selected through the comparison between each other.

(3) The proposed method on processing FLMCGDM problems can be used in many applications, such 
as the electronic-government, emergency situations and other important affairs.

(4) The proposed method also provides a kind of feasible solution for processing big data, such as the big data in social networks. And these big data solutions can be further applied into the development of personalized recommender systems.

Thus, the proposed method should be useful and effective both in theoretical and practical aspects.

\section{Acknowledgements}

This work was supported by the National Science Foundation of China (NSFC) (71171048, 71371049).

\section{References}

[1]. R. Liu, X.H. Chen, Improved clustering algorithm and its application in complex huge group decision-making, Systems Engineering \& Electronics, Vol. 28, pp. 1695-1699 (in Chinese), 2006.

[2]. S. Zahir, Clusters in a group: Decision making in the vector space formulation of the analytic hierarchy process, European Journal of Operational Research, Vol. 112, No. 3, pp. 620-634, 1999.

[3]. M. Przybyła-Kasperek and A. Wakulicz-Deja, Global decision-making system with dynamically generated clusters, Information Sciences, Vol. 270, pp. 172-191, 2014.

[4]. B. Liu, Y. Shen, X. Chen, Y. Chen, and X. Wang, A partial binary tree DEA-DA cyclic classification model for decision makers in complex multi-attribute large-group interval-valued intuitionistic fuzzy decision-making problems, Information Fusion, Vol. 18, pp. 119-130, 2014.

[5]. J.J. Zhu, S.T. Zhang, Y. Chen, and L.L. Zhang, A hierarchical clustering approach based on three-dimensional gray relational analysis for clustering a large group of decision makers with double information, Group Decision and Negotiation, Vol. 25, No. 2, pp. 325-354, 2016.

[6]. J.Q. Wang, D.D. Wang, H.Y. Zhang, and X.H. Chen, Multi-criteria group decision making method based on interval 2-tuple linguistic information and Choquet integral aggregation operators, Soft Computing, pp. 1-17, 2014.

[7]. S.M. Chen, L.W. Lee, Fuzzy multiple attributes group decision-making based on the interval type-2 TOPSIS method, Expert Systems with Applications, Vol. 37, No. 4, pp. 2790-2798, 2010.

[8]. Z.S. Xu, Q.L. Da, Combined weighted geometric averaging operator and its application, Journal of Southeast University (Natural Science Edition), Vol. 32, No. 3, pp. 506-509, 2002.

[9]. B. Liu, Y. Shen, W. Zhang, X. Chen, and X. Wang, An interval-valued intuitionistic fuzzy principal component analysis model-based method for complex multi-attribute large-group decision-making, European Journal of Operational Research, Vol. 245, No. 1, pp. 209-225, 2015.

[10]. Y.M. Wang, P. D. Liu, Multiple attribute group decision making methods based on intuitionistic linguistic power generalized aggregation operators, Applied Soft Computing, Vol. 17, pp. 90-104, 2014.

[11]. P.D. Liu, Z.M. Liu, Normal intuitionistic fuzzy Bonferroni mean operators and their applications to multiple attribute group decision making, Journal of Intelligent and Fuzzy Systems, Vol. 29, No. 5, pp. 2205-2216, 2015.

[12]. P.D. Liu, Some geometric aggregation operators based on interval intuitionistic uncertain linguistic variables and their 
application to group decision making, Applied Mathematical Modelling, Vol. 37, No. 4, pp. 2430-2444, 2013.

[13]. R.R. Yager, On ordered weighted averaging aggregation operators in multicriteria decision making, in Readings in Fuzzy Sets for Intelligent Systems. 1993, Morgan Kaufmann, pp. 80-87.

[14]. C. Hwang and F.C.H. Rhee, Uncertain fuzzy clustering: interval type-2 fuzzy approach to c-means, IEEE Transactions on Fuzzy Systems, Vol. 15, No. 1, pp. 107-120, 2007.

[15]. G.S. Liang, T.Y. Chou, and T.C. Han, Cluster analysis based on fuzzy equivalence relation, European Journal of Operational Research, Vol. 166, No. 1, pp. 160-171, 2005.

[16]. J.T. Zhang and M. Li. Self-organizing feature map clustering based on fuzzy equivalence relation and its application in ecological analysis. in 2011 Eighth International Conference on Fuzzy Systems and Knowledge Discovery (FSKD). 2011.

[17]. A.B. Raut and G.R. Bamnote. Clustering method based on fuzzy equivalence relation. in Computer and Communication Technology (ICCCT), 2011 2nd International Conference on. 2011.

[18]. S. Kumar, M. Kathuria, A.K. Gupta, and M. Rani. Fuzzy clustering of web documents using equivalence relations and fuzzy hierarchical clustering. in 2012 CSI Sixth International Conference on Software Engineering (CONSEG). 2012.

[19]. Y.J. Wang, A clustering method based on fuzzy equivalence relation for customer relationship management, Expert Systems with Applications, Vol. 37, No. 9, pp. 6421-6428, 2010.

[20]. A. Yazdani-Chamzini, An integrated fuzzy multi criteria group decision making model for handling equipment selection, Journal of Civil Engineering and Management, Vol. 20, No. 5, pp. 660-673, 2014.

[21]. X.J. Wang and H.K. Chan, A hierarchical fuzzy TOPSIS approach to assess improvement areas when implementing green supply chain initiatives, International Journal of Production Research, Vol. 51, No. 10, pp. 3117-3130, 2013.

[22]. T.C. Wang and Y.H. Chen, Fuzzy multi-criteria selection among transportation companies with fuzzy linguistic preference relations, Expert Systems with Applications, Vol. 38, No. 9, pp. 11884-11890, 2011.

[23]. R.A. Krohling and V.C. Campanharo, Fuzzy TOPSIS for group decision making: A case study for accidents with oil spill in the sea, Expert Systems with Applications, Vol. 38, No. 4, pp. 4190-4197, 2011.

[24]. C.T. Chen, Extensions of the TOPSIS for group decision-making under fuzzy environment, Fuzzy Sets and Systems, Vol. 114, No. 1, pp. 1-9, 2000

[25]. J.M. Mendel, Computing with words: Zadeh, Turing, Popper and Occam, IEEE Computational Intelligence Magazine, Vol. 2, No. 4, pp. 10-17, 2007.

[26]. L.A. Zadeh, The concept of a linguistic variable and its application to approximate reasoning-I, Information Sciences, Vol. 8, No. 3, pp. 199-249, 1975.

[27]. Y.B. Gong, Fuzzy multi-attribute group decision making method based on interval type-2 fuzzy sets and applications to global supplier selection, International Journal of Fuzzy Systems, Vol. 15, No. 4, pp. 392-400, 2013.

[28]. J.D. Qin, X.W Liu, and W. Pedrycz, An extended VIKOR method based on prospect theory for multiple attribute decision making under interval type-2 fuzzy environment, Knowledge-Based Systems, Vol. 86, pp. 116-130, 2015.

[29]. J.D Qin, X.W Liu, and W. Pedrycz, A multiple attribute interval type-2 fuzzy group decision making and its application to supplier selection with extended LINMAP method, Soft Computing, pp. 1-20, 2016.

[30]. M. Pratama, J. Lu, G Zhang, Evolving type-2 fuzzy classifier, IEEE Transactions on Fuzzy Systems, Vol. 24, No. 3, pp. 
574-589, 2016.

[31]. T. Wu, X.W. Liu. X.Z. Sang, Clustering analysis of fuzzy equivalence based on interval type-2 fuzzy sets, Systems Engineering--Theory \& Practice, Vol. 36, No. 1, pp. 1-10 (in Chinese), 2016.

[32]. J. Aczél and T.L. Saaty, Procedures for synthesizing ratio judgements, Journal of Mathematical Psychology, Vol. 27, No. 1, pp. 93-102, 1983.

[33]. Z.S. Xu, Q.L. Da, The ordered weighted geometric averaging operators, International Journal of Intelligent Systems, Vol. 17, pp. 709-716, 2002.

[34]. Y.C. Dong, H.J. Zhang, and E. Herrera-Viedma, Consensus reaching model in the complex and dynamic MAGDM problem, Knowledge-Based Systems, Vol. 106, pp. 206-219, 2016.

[35]. Z.W. Gong, H.H. Zhang, J. Forrest, L.S. Li, and X.X. Xu, Two consensus models based on the minimum cost and maximum return regarding either all individuals or one individual, European Journal of Operational Research, Vol. 240, No. 1, pp. 183-192, 2015.

[36]. I. Palomares, L. Martinez, and F. Herrera, A consensus model to detect and manage noncooperative behaviors in large-scale group decision making, IEEE Transactions on Fuzzy Systems, Vol. 22, No. 3, pp. 516-530, 2014.

[37]. X.H. Xu, Z.J. Du, and X.H. Chen, Consensus model for multi-criteria large-group emergency decision making considering non-cooperative behaviors and minority opinions, Decision Support Systems, Vol. 79, pp. 150-160, 2015.

[38]. F. Zhang, et al., An improved consensus-based group decision making model with heterogeneous information, Applied Soft Computing, Vol. 35, pp. 850-863, 2015.

[39]. B.S. Liu, Y.H. Shen, W. Zhang, X.H. Chen, and X.Q. Wang, An interval-valued intuitionistic fuzzy principal component analysis model-based method for complex multi-attribute large-group decision-making, European Journal of Operational Research, Vol. 245, No. 1, pp. 209-225, 2015.

[40]. J.M. Mendel, R.I.B. John, F.L. Liu, Interval type-2 fuzzy logic systems made simple, IEEE Transactions on Fuzzy Systems, Vol. 14, No. 6, pp. 808-821, 2006.

[41]. J.M. Mendel, R.I.B. John, Type-2 fuzzy sets made simple, IEEE Transactions on Fuzzy Systems, Vol. 10, No. 2, pp. 117-127, 2002.

[42]. D.R. Wu, J.M. Mendel, A comparative study of ranking methods, similarity measures and uncertainty measures for interval type-2 fuzzy sets, Information Sciences, Vol. 179, No. 8, pp. 1169-1192, 2009.

[43]. N.N. Karnik, J.M. Mendel, Operations on type-2 fuzzy sets, Fuzzy Sets and Systems, Vol. 122, pp. 327-348, 2001. 